\title{
Enhancing Entangled Two-Photon Absorption for Picosecond Quantum Spectroscopy
} Ryan K. Burdick ${ }^{\dagger}$, George C. Schatz ${ }^{\ddagger}$, Theodore Goodson III $^{\dagger *}$

${ }^{\dagger}$ Department of Chemistry, University of Michigan, Ann Arbor, MI 48109-1055, United States

${ }^{\ddagger}$ Department of Chemistry, Northwestern University, Evanston, IL 60208-3113, United States

*tgoodson@umich.edu

\section{Supporting Information}

\section{Calculation of entanglement time with dispersion effects}

Since $\sigma_{f}$ was always smaller than the initial SPDC bandwidth emitted by the crystal $\left(\sigma_{L}\right)$, $T_{e}$ was calculated in Fig. $1 \mathrm{~b}$ of the manuscript from $\sigma_{f}$, taking dispersion into account using the standard relation for the pulse width in a dispersive medium: ${ }^{1}$

$$
T_{e}=\frac{1}{\sigma_{f}} \sqrt{1+\left(4 \ln 2 \frac{G D D}{\left(\frac{1}{\sigma_{f}}\right)^{2}}\right)^{2}}
$$

$G D D$ in this expression is the group delay dispersion for the optical elements in our experiment.

Origin and derivation of entanglement area as a function of entanglement time

When the location of the signal photon in the transverse plane is measured, the transverse location of the idler photon is also known to within an uncertainty determined by the entanglement area, $A_{e}{ }^{2}$ The origin of $A_{e}$ is an uncertainty in the emission angles of the idler photon from the SPDC crystal, shown in Fig. S1. 3,4 


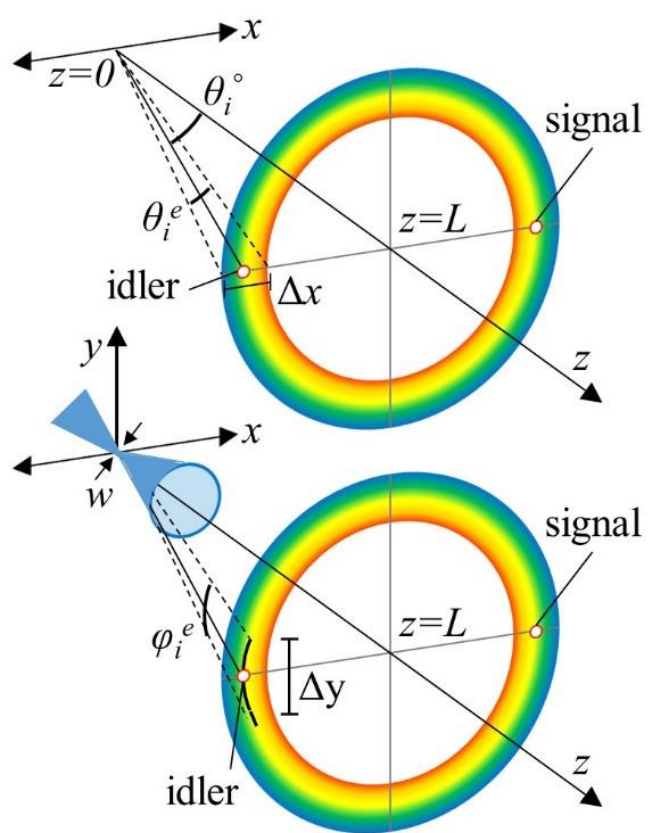

Fig. S1. Emission angles and their uncertainties of Type-I SPDC from a crystal with length $L$ along the pump beam propagation axis (z-axis).

The idler photon is emitted in the x-z plane at the central polar angle $\theta_{i}^{\circ}$ with an uncertainty, or polar entanglement angle, $\theta_{i}{ }^{e}$. The polar entanglement angle creates uncertainty of the photon location in the $\mathrm{x}-\mathrm{z}$ plane, $\Delta x$. The uncertainty in the $\mathrm{y}$-direction, $\Delta y$, comes from the azimuthal entanglement angle, $\varphi_{i}{ }^{e}$. This angle originates from the divergence angle of the focused pump beam with waist $w . A_{e}$ is defined by the product of uncertainties $\Delta x$ and $\Delta y .^{2}$

While the azimuthal entanglement angle is easily seen from the pump divergence angle, where $\Delta y$ is proportional to $1 / w$, the polar entanglement angle has a more complicated origin. When SPDC photons are created inside of the crystal, they are in a superposition of frequencies, which originates from the phase mismatch of the pump and SPDC photons' wave vectors along the z-axis, $\Delta k_{z}$. It is well known that expanding $\Delta k_{z}$ as a Taylor series results in the approximation: ${ }^{5}$

$$
\Delta k_{z} \approx-v D-\frac{v^{2}}{2} D^{\prime \prime}
$$

Here, $v$ is the detuning frequency from the central frequency of the SPDC photons, $D$ is the difference in inverse group velocity of the signal and idler, and $D$ ' 'is the group velocity dispersion (GVD) of the photons. Higher order terms in $v$ are negligible. For Type-II SPDC (and nondegenerate Type-I/-0 SPDC), the signal and idler have different group velocities, so $D$ is non-zero, and the second-order term is negligible. For degenerate Type-I/-0 SPDC (which we will refer to as "Type-I" from here for brevity), the signal and idler have the same group velocity, so $D$ vanishes and only the second-order term is left. Thus, the phase mismatch for Type-II SPDC depends linearly on the SPDC frequency, while the phase mismatch for Type-I SPDC depends quadratically on the SPDC frequency.

For Type-II SPDC, each frequency of the idler photon travels at a different polar angle inside the crystal due to the different refractive indices. Thus, the frequencies are spread out in space when they exit the crystal. Because the idler has an uncertainty in its frequency (the bandwidth of its frequency superposition), the photon also has an uncertainty in its polar emission 
angle, i.e. the polar entanglement angle. Decreasing the frequency uncertainty also reduces the polar entanglement angle linearly. For Type-I SPDC, each frequency of the idler is dispersed inside the crystal due to GVD. The GVD, which is second-order dispersion, then determines the uncertainty in the polar emission angle. Reducing the frequency uncertainty thus decreases the polar entanglement angle quadratically.

One way to change the polar entanglement angle is by changing the crystal length, $L$, which was shown in a paper from Joobeur et. al. ${ }^{3}$ They showed that by increasing $L$, the polar entanglement angle decreases, which would decrease $A_{e}$. Increasing $L$ has the effect of decreasing the SPDC bandwidth, and so decreasing the bandwidth should also decrease the polar entanglement angle and $A_{e}{ }^{5}$ In our experiments, we keep $L$ constant and decrease the SPDC bandwidth with frequency bandpass filters and a monochromator. For Type-I SPDC, since the frequency uncertainty is dependent on second-order dispersion, the uncertainty should be related to the filter or monochromator's bandwidth $\left(\sigma_{f}\right)$ squared. The relationship between $A_{e}$ and the bandwidth can be represented simply (taking the $\Delta y$ contribution to be independent of $\sigma_{f}$ ) as:

$$
A_{e} \propto \theta_{i}^{e} \propto \alpha \sigma_{f}^{2}+A_{d}
$$

$\alpha$ is a coefficient to be solved for by fitting experimental results, and here we include a constant parameter $A_{d}$ in this formula to account for the fact that $A_{e}$ cannot be smaller (at least for classical light) than the diffraction limited area. As mentioned in the main manuscript, $A_{d}$ should be a number on the order of $(\lambda / 2)^{2}=1.6 \times 10^{-9} \mathrm{~cm}^{2}$.

While eq. $\mathrm{S} 3$ shows that $A_{e}$ decreases as $\sigma_{f}$ decreases, it also suggests that $A_{e}$ would increase as long as $\sigma_{f}$ continues to increase. This latter point, however, is not physically realistic since the SPDC has an initial bandwidth determined by the SPDC crystal length before the photons are filtered further downstream. The SPDC bandwidth, and thus $A_{e}$, cannot be bigger than that initially formed in the SPDC crystal. We can account for this upper limit with the following phenomenological modification to eq. S3:

$$
A_{e} \propto \theta_{i}^{e} \propto\left(\frac{\alpha}{\sigma_{f}^{2}}+\frac{\beta}{\sigma_{L}^{2}}\right)^{-1}+A_{d}
$$

$\sigma_{L}$ is the SPDC bandwidth defined by the crystal with length $L$, and it has a coefficient $\beta$ to be solved for. Eq. S4 is a simple way to represent that when $\sigma_{f} \ll<\sigma_{L}$, the second term is negligible, and $A_{e}$ is determined by the filter but subject to a diffraction limit. When $\sigma_{f} \gg>\sigma_{L}$, the first term is negligible, and $A_{e}$ is determined by the SPDC crystal. Eq. S4 was used in eq. 1 of the main text to fit with the Type-I experimental data in Fig. 1 of the main text. The coefficients $\alpha$ and $\beta$ were the fitting parameters. With the coefficients, $A_{e}$ was calculated with eq. S4, and the upper and lower bounds in our experiment are reported in the main text.

For Type-II SPDC, the frequency uncertainty is determined by first-order dispersion, and thus is related to the SPDC bandwidth linearly. Following along the same lines as eq. S3-4 for Type-I, but keeping $A_{e}$ proportional to the frequency bandwidth linearly, a similar expression for $A_{e}$ when using Type-II SPDC can be used:

$$
A_{e} \propto \theta_{i}^{e} \propto\left(\frac{\gamma}{\sigma_{f}}+\frac{\zeta}{\sigma_{L}}\right)^{-1}+A_{d}
$$


$\gamma$ and $\zeta$ are the coefficients, which were calculated by using eq. S5 in eq. 1 of the main text and fitting with the Type-II experimental data in Fig. 1 of the main text. The upper and lower $A_{e}$ are reported in the main text.

Using the fitted parameters for eq. S5, the range of $A_{e}$ from its minimum to maximum value for both SPDC Types are plotted in Fig. S2.

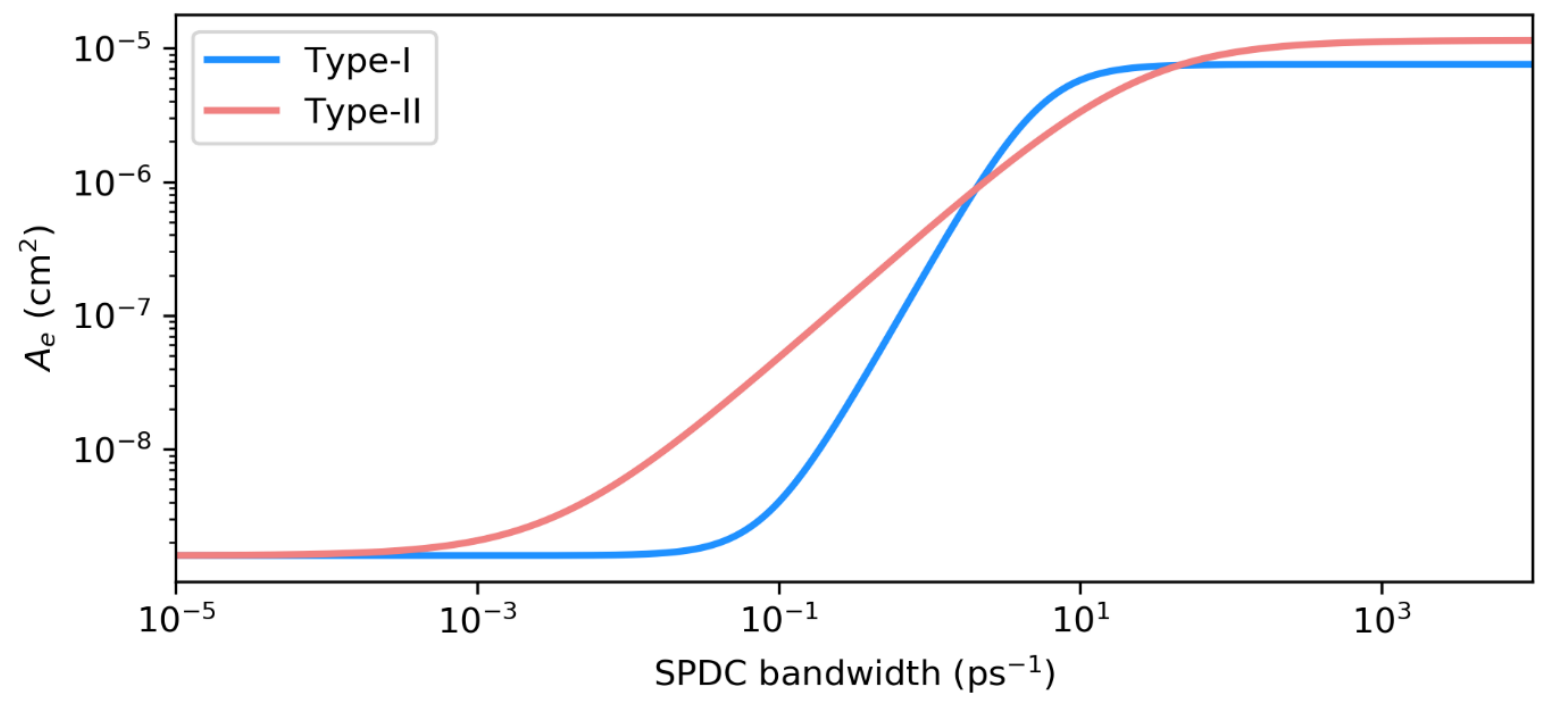

Figure S2. $A_{e}$ vs the SPDC bandwidth for Type-I and Type-II SPDC.

ETPA cross-section vs $T_{e}$ for sinc and Gaussian function SPDC spectra with and without dispersion

The ETPA cross-section vs $T_{e}$ was calculated using eq. 1 from the main text for three cases: increasing $A_{e}$ with increasing $\sigma_{f}$ using eq. S4 (S5) for Type-I (Type-II) SPDC, constant $A_{e}$ that has been assumed historically, and decreasing $A_{e}$ for comparison. In the calculation, we use the model of a strong TPA chromophore. ${ }^{6}$ We consider the Q band in ZnTPP at $550 \mathrm{~nm}$ as the most dominant intermediate state due to its smallest detuning energy. ${ }^{7}$ Calculating the linewidth of intermediate excited states in molecules can be very challenging, so using an upper limit of $k_{j}=0.1 \mathrm{eV}$ for organic chromophores in solution is reasonable and standard practice. ${ }^{8}$ The TDM product in eq. 1 was estimated to be $16.1 \mathrm{D}^{2}$ using the classical TPA cross-section of ZnTPP and the classical TPA cross-section equation. ${ }^{9,10}$ These parameters were used for the calculations in Fig. 1-2 of the main text and Fig. S3-S4. In Fig. S3, a Gaussian SPDC spectrum was used, and both the cases with and without dispersion were used to calculate $T_{e}$ (eq. 3 of the main text) are shown. The sinc spectra (Fig. S4) yield the well-known oscillations when $T_{e}<\sim 500 \mathrm{fs},{ }^{11}$ but the overall trend as $T_{e}$ increases is the same regardless of the SPDC spectral function. Two important features of Figs. S3 and S4 are: (1) For the results that include dispersion, there are two branches to the cross-sections (the upper branch corresponding to small $\sigma_{f}$ and the lower branch for high $\sigma_{f}$ ) when plotted as a function of $T_{e}$ for both Type-I and Type-II, with the two branches coming together at $T_{e}$ slightly below 100 fs; (2) For the upper branch, the Type-I cross-section increases linearly with increasing $1 / \sigma_{f}$ as $\sigma_{f}$ goes toward $0.1 \mathrm{ps}^{-1}$, where $A_{e}$ is limited by the diffraction area $A_{d}$. At this bandwidth, the Type-I cross-section reaches a maximum, and the Type-II cross-section plateaus before decreasing. 

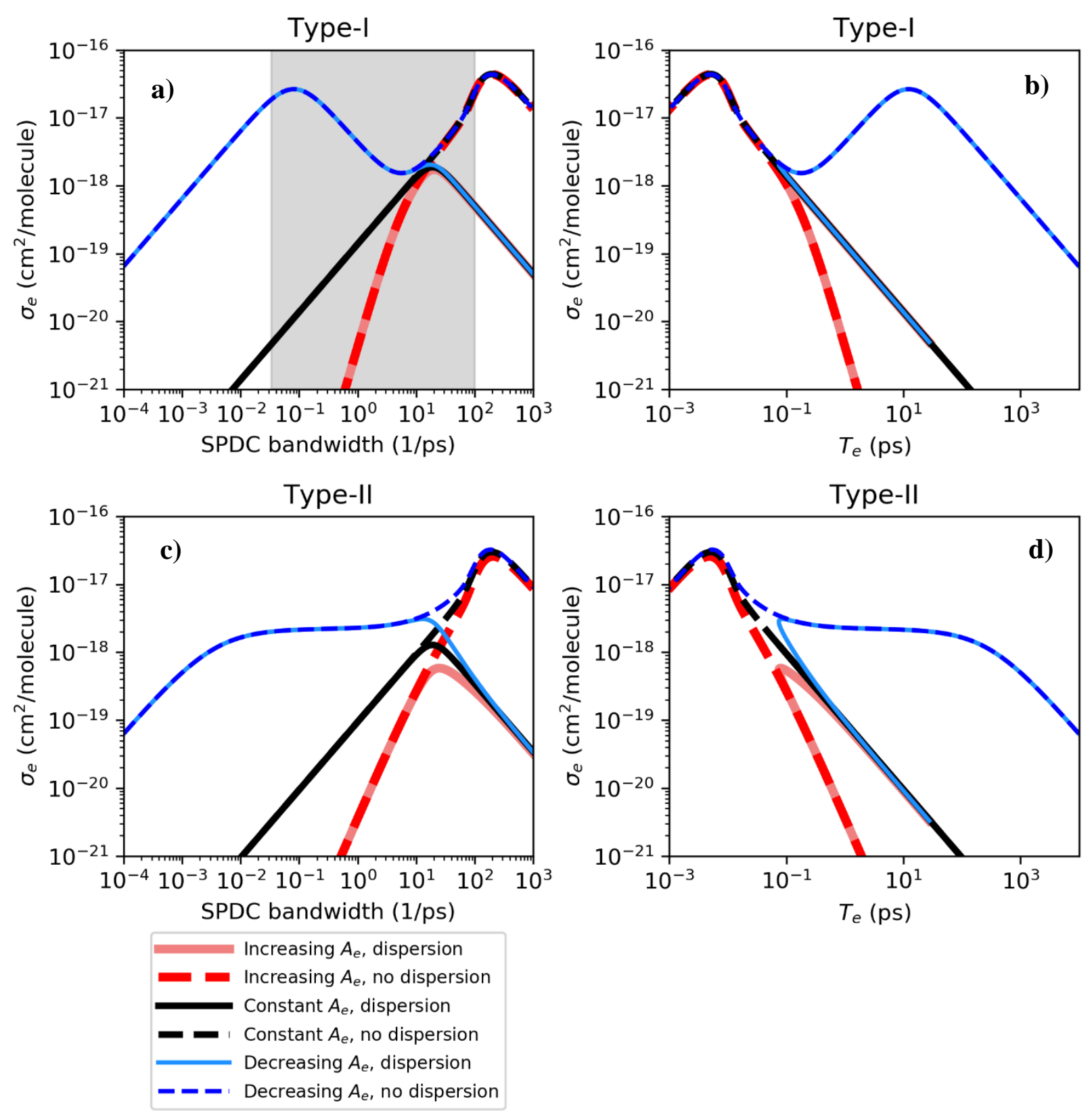

Figure S3. a) Type-I ETPA cross-section, $\sigma_{e}$, vs $\sigma_{f}$ using Gaussian SPDC spectra for increasing $A_{e}$ (red), constant $A_{e}$ (black), and decreasing $A_{e}$ (blue) with (solid) and without (dotted) dispersion. The shaded region is the bandwidth range that is shown in Fig. 2 of the main text; b) Same as (a) but with the X-axis converted to $T_{e}$; c) Type-II $\sigma_{e}$ vs $\sigma_{f}$ using Gaussian SPDC spectra for increasing $A_{e}$ (red), constant $A_{e}$ (black), and decreasing $A_{e}$ (blue) with (solid) and without (dotted) dispersion. d) Same as (c) but with the x-axis converted to $T_{e}$; The same parameters from Fig. 1 of the main text are used here. 

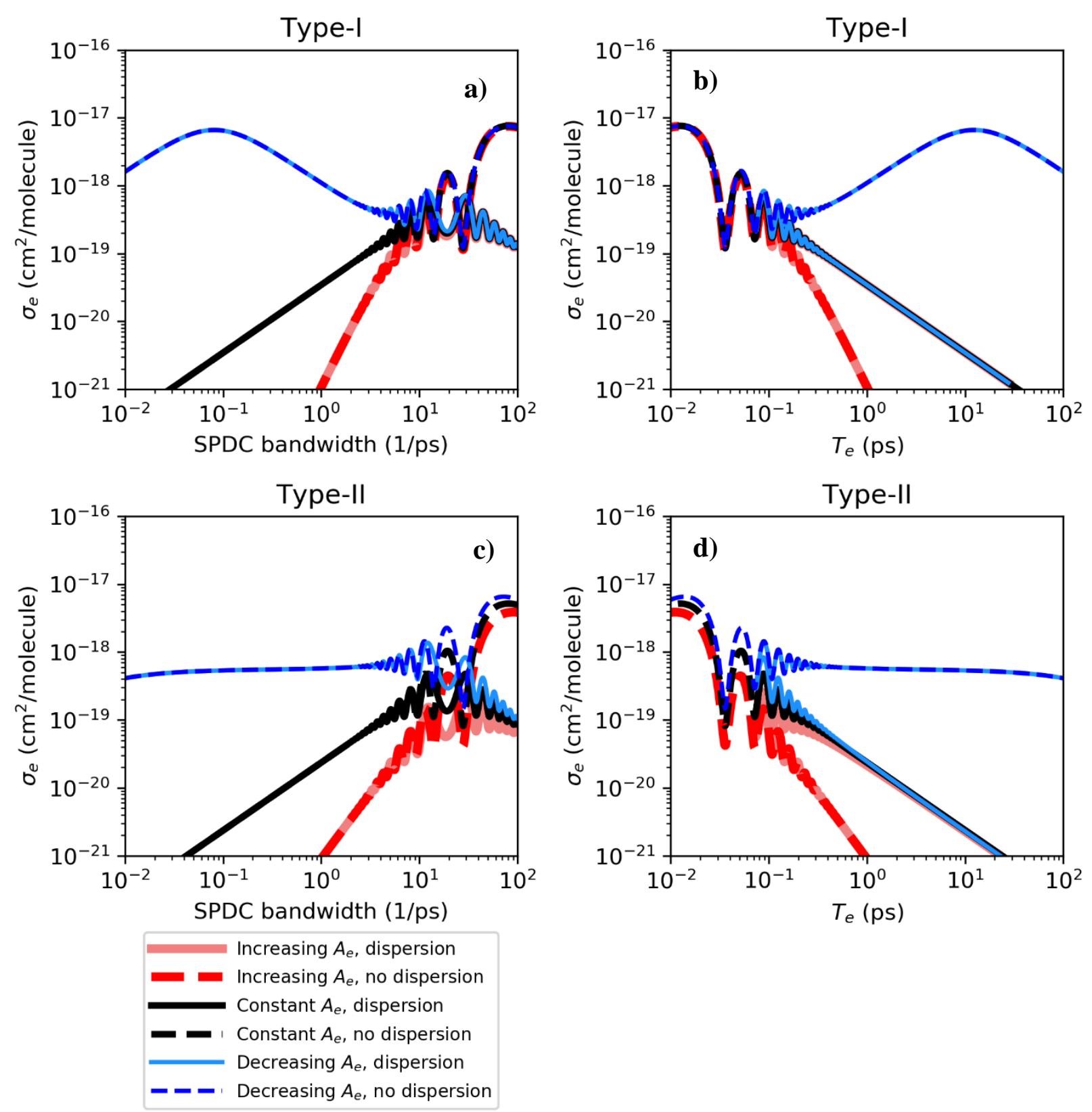

Figure S4. a) Type-I ETPA cross-section, $\sigma_{e}$, vs $\sigma_{f}$ using sinc SPDC spectra for increasing $A_{e}$ (red), constant $A_{e}$ (black), and decreasing $A_{e}$ (blue) with (solid) and without (dotted) dispersion. The shaded region is the bandwidth range that is shown in Fig. 2 of the main text; b) Same as (a) but with the X-axis converted to $T_{e}$; c) Type-II $\sigma_{e}$ vs $\sigma_{f}$ using sinc SPDC spectra for increasing $A_{e}$ (red), constant $A_{e}$ (black), and decreasing $A_{e}$ (blue) with (solid) and without (dotted) dispersion. d) Same as (c) but with the $\mathrm{x}$-axis converted to $T_{e}$; The same parameters from Fig. 1 of the main text are used here.

\section{Experimental details}

Our experimental setup uses a $405 \mathrm{~nm}$ CW laser with maximum SPDC pump power of $\sim 150 \mathrm{~mW}$. The pump beam is focused into a Type-I or Type-II SPDC crystal cut for degenerate, non-collinear phase-matching. After removing the residual pump beam with a dichroic mirror and 
longpass filters, the SPDC is sent through one of several bandpass filters with different bandwidths $(10,30$, and $80 \mathrm{~nm})$ or a monochromator with variable bandwidth $(2,2.5,3,4$, and $5 \mathrm{~nm})$ to alter the SPDC spectral bandwidth. After filtering, the SPDC is focused into a cuvette, and the transmitted entangled pairs are separated with a knife-edge mirrored prism. Coincidence counts were measured with single-photon detecting APDs with a 5 ns coincidence window. The difference in the transmitted coincidence counts for the cuvette filled with pure solvent vs the sample solution ( $20 \mu \mathrm{M}$ zinc tetraphenylporphyrin, ZnTPP, in toluene) gives the ETPA rate. Calculation of the ETPA cross-section from this rate was completed as reported in previous works: ${ }^{9,12}$

$$
\sigma_{e}=\frac{m}{l c N_{A}}
$$

$m$ is the slope of the linear fit for ETPA rate vs input rate, $l$ is the cuvette length, $c$ is the concentration, and $N_{A}$ is Avogradro's number. The plots of ETPA rate in ZnTPP vs input rate are given in Fig. S5-6. Since reducing the SPDC bandwidth also reduces the input rate in a passive filtering scheme used here, the input rates were normalized for each bandwidth for easy comparison of the slopes. The y-axis was normalized so that the slope for each bandwidth is the same as what it was before normalization.

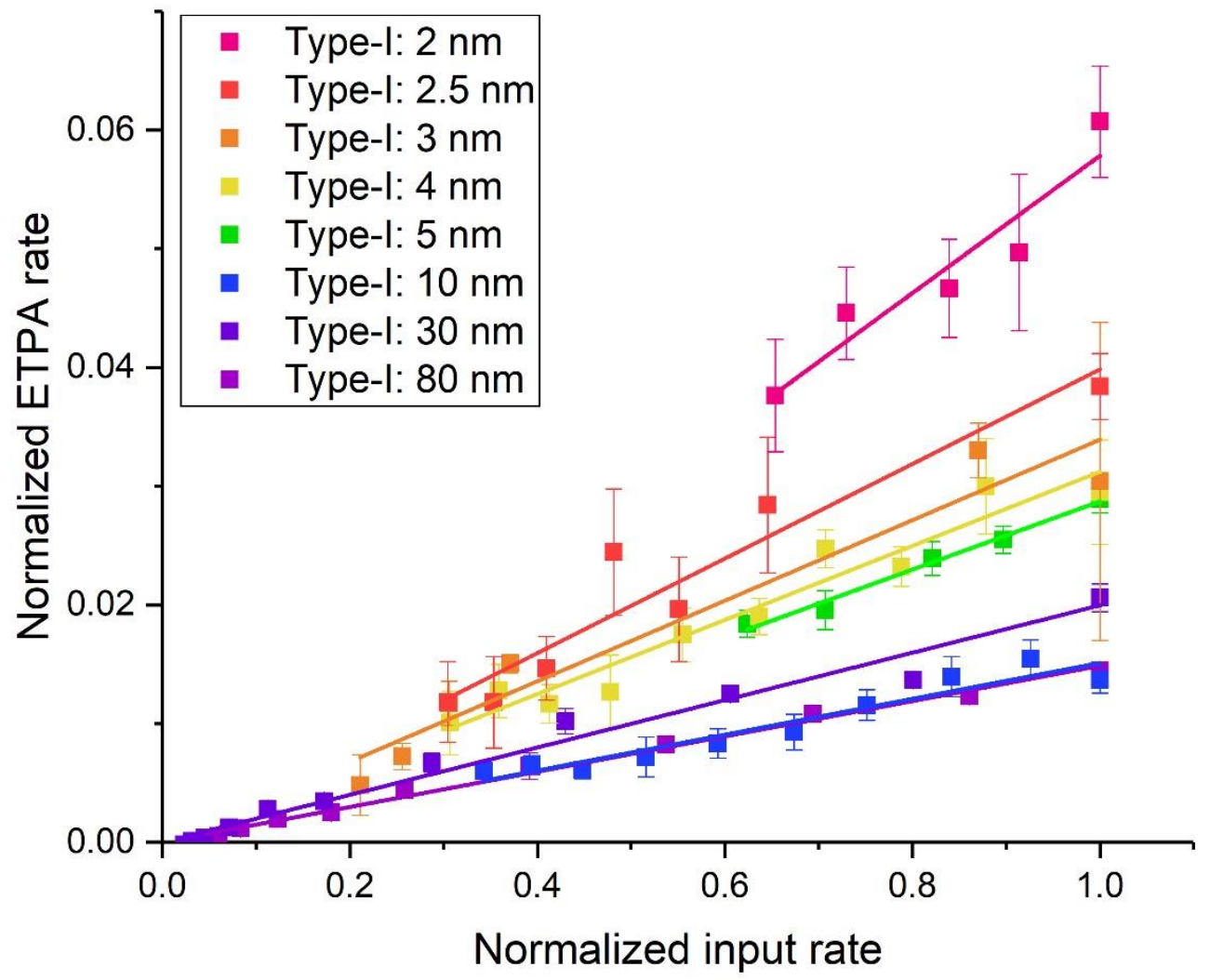

Figure S5. Type-I ETPA rate in ZnTPP vs input rate for all SPDC bandwidths. 


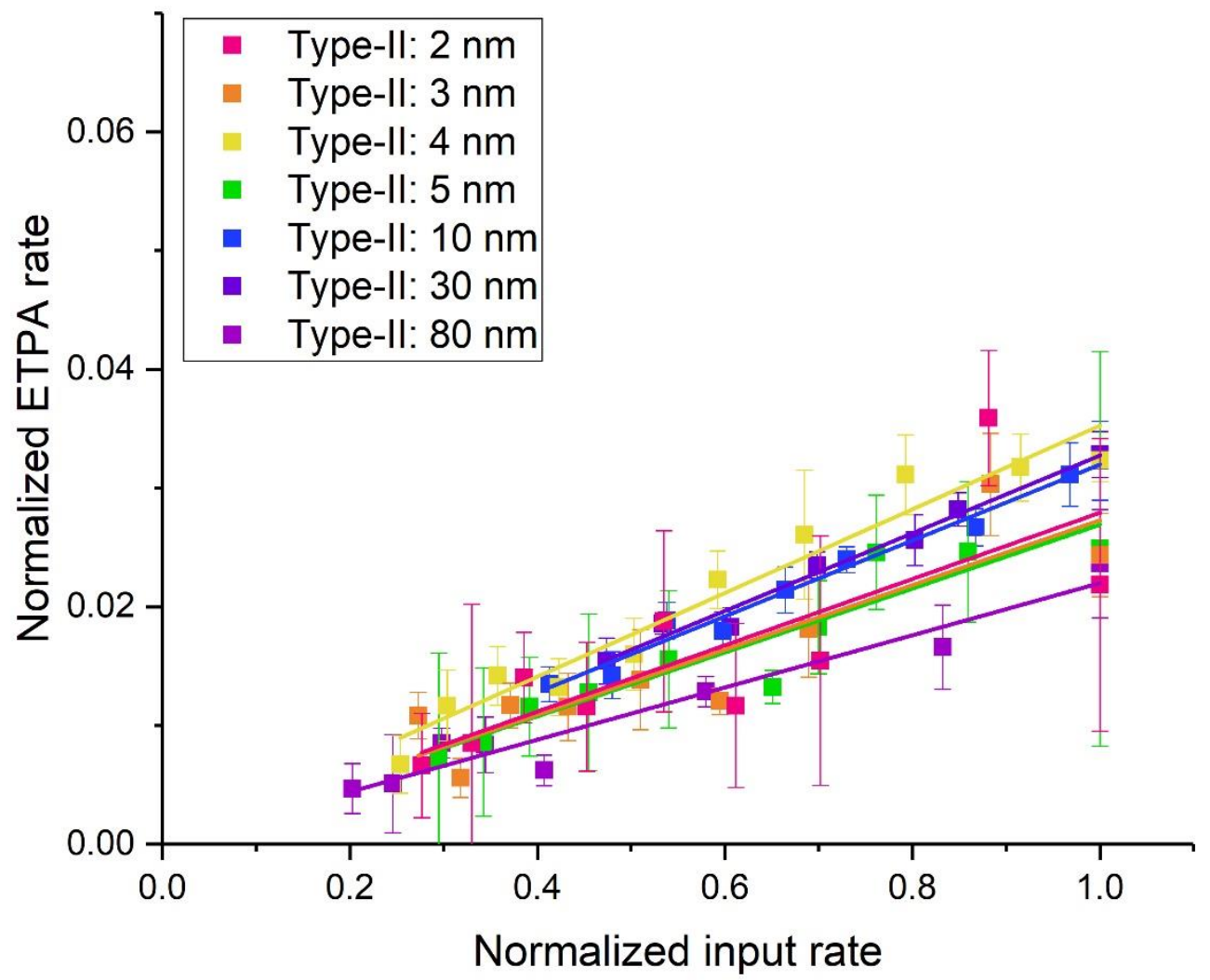

Figure S6. Type-II ETPA rate in ZnTPP vs input rate for all SPDC bandwidths.

The dependence of the SPDC attenuation percentage (slope of ETPA rate vs input rate) on the SPDC bandwidth, or $T_{e}$, shows that the SPDC attenuation is due to ETPA and not other linear optical processes, such as one-photon absorption or scattering. These other optical processes would not depend on $T_{e}$. Additionally, the different ETPA cross-section dependences vs $\sigma_{f}$ (or $T_{e}$ ) for Type-I vs Type-II SPDC also show that the measured signal is not due to other optical processes since for a one-photon process, Type-I and Type-II SPDC only differ in the polarizations of the photons. The molecule being in solution would rotationally average out any polarization dependence of a one-photon optical process. Thus, if a one-photon process were attenuating the SPDC, the Type-I and Type-II SPDC would have yielded the same ETPA cross-section with no dependence on $\sigma_{f}$ or $T_{e}$. This is clearly not the case in our measurements.

\section{ETPA cross-section measurements in flavin adenine dinucleotide}

To ensure that the ETPA cross-section vs $\sigma_{f}$ trends we measured in ZnTPP for Type-I and Type-II SPDC are indicative of the SPDC properties and not something unique to ZnTPP, we performed similar measurements in another molecule that has been studied with ETPA: flavin adenine dinucleotide (FAD). ${ }^{12}{ }^{13}$ Fig. S7-S8 shows the normalized ETPA rate vs input rate plots 
for the cross-sections for FAD. We obtained the same cross-section trends for Type-I and Type-II ETPA with FAD, shown in Fig. S9, validating our proposed theory that changing $\sigma_{f}$ is also changing $A_{e}$, independent of the chromophore.

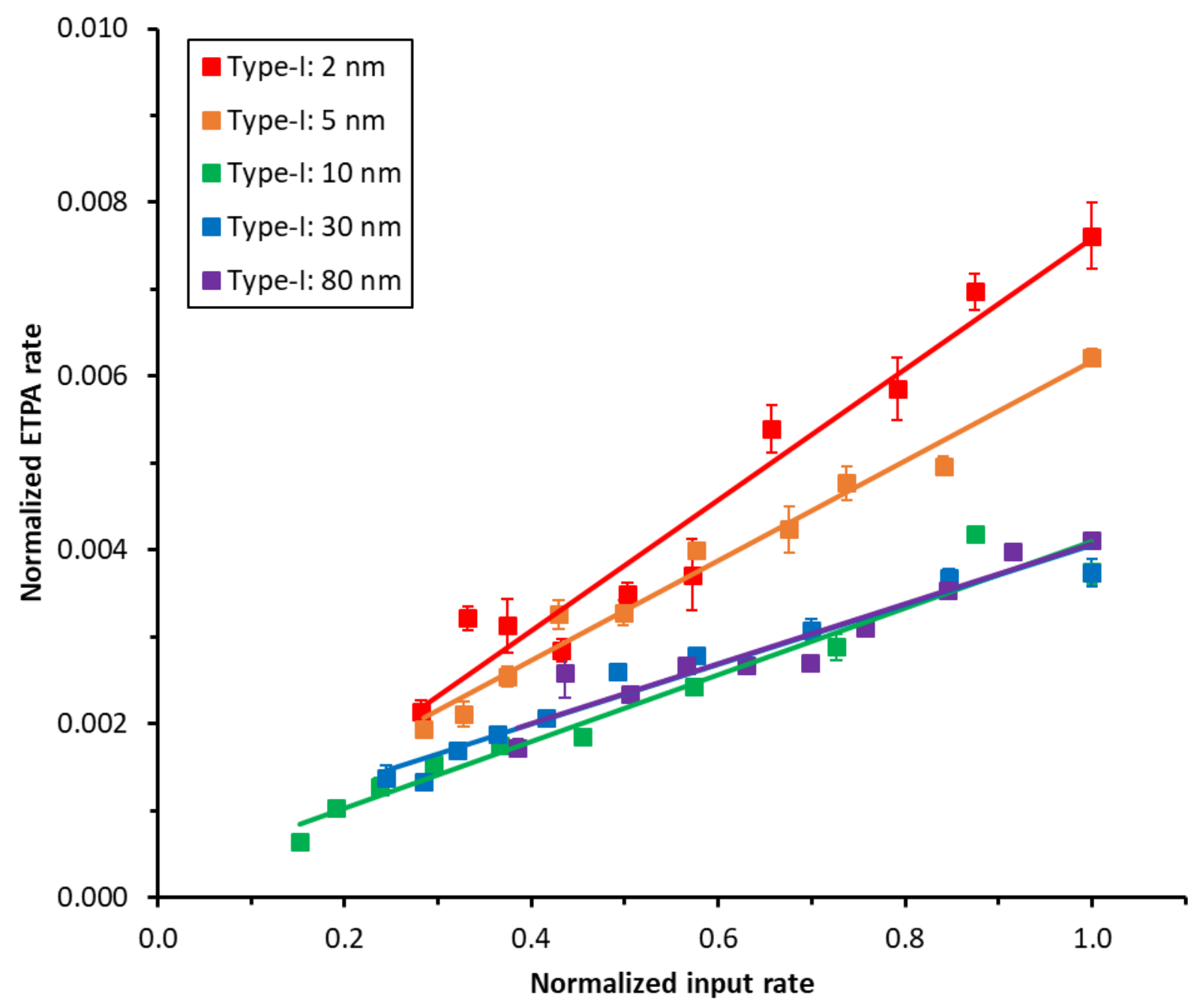

Figure S7. Type-I ETPA rate vs input rate in FAD for all SPDC bandwidths. 


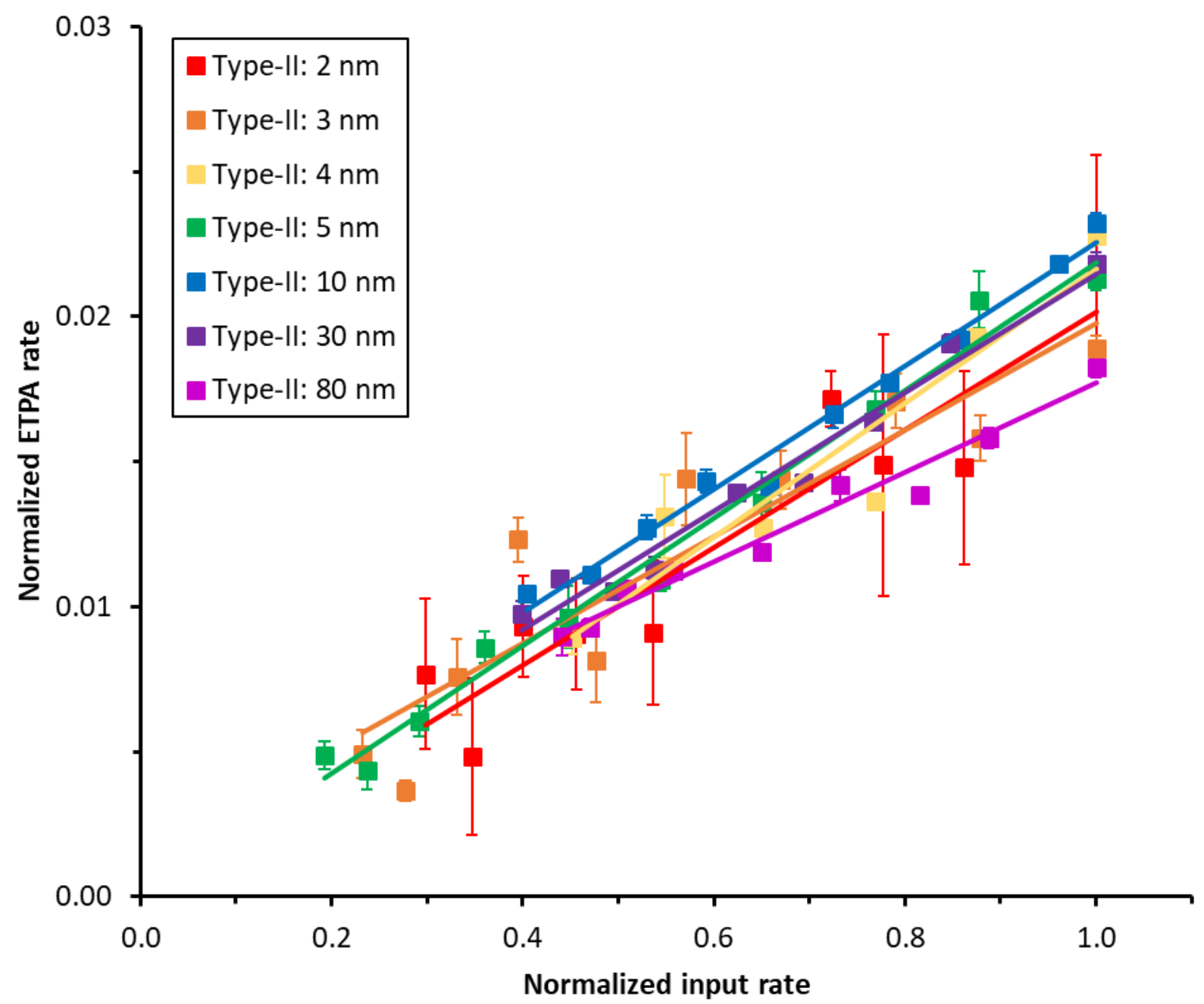

Figure S8. Type-II ETPA rate vs input rate in FAD for all SPDC bandwidths. 

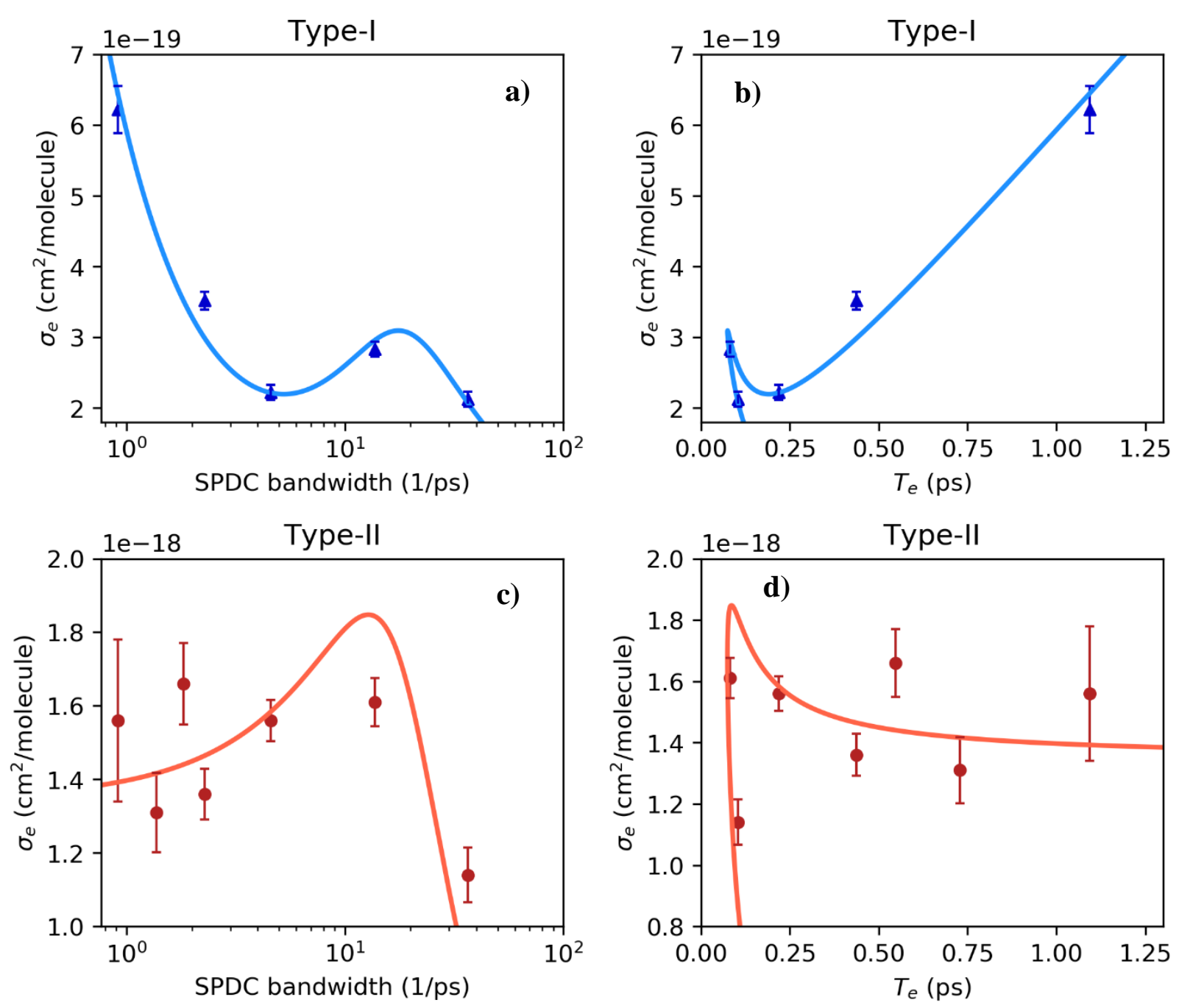

Figure S9. a) Experimental (points) and theoretical (solid curve) Type-I ETPA cross-sections, $\sigma_{e}$, vs $\sigma_{f}$ for FAD, with dispersion applied. b) Same as (a) but with the x-axis converted to $T_{e}$. c) Experimental (points) and theoretical (solid curve) Type-II $\sigma_{e}$ vs $\sigma_{f}$ for FAD, with dispersion applied. d) Same as (c) but with the x-axis converted to $T_{e}$.

\section{Effects of vibrational relaxation and of molecular motion.}

Vibrational relaxation could also cause the modulus squared term to decrease as $T_{e}$ increases if eq. 1 included the vibrational wave function of the molecule. However, assuming that the vibrational states have an exponential decay like the electronic states, the modulus squared term would have a Lorentzian function dependent on the vibrational lifetime. The tails of a Lorenztian decrease slower than the plasma dispersion function which defines the electronic state relaxation in eq. 1, so the vibrational lifetime would not cause the light-matter coupling to decrease any faster than it is already decreasing from the electronic lifetime. As a result, the trend of the ETPA cross-section vs $T_{e}$ should hardly be affected by vibrations. This same reasoning applies to the rotational movement of the molecule. 


\section{References}

1. Newport Corporation. The Effect of Dispersion on Ultrashort Pulses. https://www.newport.com/n/the-effect-of-dispersion-on-ultrashort-pulses (accessed 2021-04-19).

2. Peřina, J.; Saleh, B. E. A.; Teich, M. C., Multiphoton absorption cross section and virtual-state spectroscopy for the entangled \$n\$-photon state. Physical Review A 1998, 57 (5), 3972-3986.

3. Joobeur, A.; Saleh, B. E. A.; Larchuk, T. S.; Teich, M. C., Coherence properties of entangled light beams generated by parametric down-conversion: Theory and experiment. Physical Review A 1996, 53 (6), 4360-4371.

4. Joobeur, A.; Saleh, B. E. A.; Teich, M. C., Spatiotemporal coherence properties of entangled light beams generated by parametric down-conversion. Physical Review A 1994, 50 (4), 3349-3361.

5. Yoon-Ho, K., Measurement of one-photon and two-photon wave packets in spontaneous parametric downconversion. Journal of the Optical Society of America B 2003, 20 (9), 19591966.

6. Lahankar, S. A.; West, R.; Varnavski, O.; Xie, X.; Goodson, T.; Sukhomlinova, L.; Twieg, R., Electronic interactions in a branched chromophore investigated by nonlinear optical and time-resolved spectroscopy. The Journal of Chemical Physics 2003, 120 (1), 337-344.

7. Barnett, G. H.; Hudson, M. F.; Smith, K. M., Concerning meso-tetraphenylporphyrin purification. Journal of the Chemical Society, Perkin Transactions 1 1975, (14), 1401-1403.

8. Kang, G.; Nasiri Avanaki, K.; Mosquera, M. A.; Burdick, R. K.; Villabona-Monsalve, J. P.; Goodson Iii, T.; Schatz, G. C., Efficient modeling of organic chromophores for entangled two-photon absorption. Journal of the American Chemical Society 2020.

9. Upton, L.; Harpham, M.; Suzer, O.; Richter, M.; Mukamel, S.; Goodson, T., Optically Excited Entangled States in Organic Molecules Illuminate the Dark. Journal of Physical Chemistry Letters 2013, 4 (12), 2046-2052.

10. Burdick, R. K.; Varnavski, O.; Molina, A.; Upton, L.; Zimmerman, P.; Goodson, T., Predicting and Controlling Entangled Two-Photon Absorption in Diatomic Molecules. The Journal of Physical Chemistry A 2018, 122 (41), 8198-8212.

11. Fei, H.-B.; Jost, B. M.; Popescu, S.; Saleh, B. E. A.; Teich, M. C., EntanglementInduced Two-Photon Transparency. Physical Review Letters 1997, 78 (9), 1679-1682.

12. Villabona-Monsalve, J. P.; Burdick, R. K.; Goodson, T., Measurements of Entangled Two-Photon Absorption in Organic Molecules with CW-Pumped Type-I Spontaneous Parametric Down-Conversion. The Journal of Physical Chemistry C 2020, 124 (44), 2452624532.

13. Villabona-Monsalve, J. P.; Varnavski, O.; Palfey, B. A.; Goodson, T. I., Two-Photon Excitation of Flavins and Flavoproteins with Classical and Quantum Light. J. Am. Chem. Soc. 2018, 140 (44), 14562-14566. 